\title{
GEOMETRY RELAXATION OF PHOTOEXCITED STATES IN CONJUGATED MOLECULES
}

\author{
S. TRETIAK* ${ }^{*}$ A. SAXENA, R.L. MARTIN and A.R. BISHOP \\ Theoretical Division and Center for Nonlinear Studies, \\ Los Alamos National Laboratory, Los Alamos, New Mexico 87545
}

(Received 14 November 2001)

\begin{abstract}
The random phase approximation combined with semiempirical Hamiltonians is applied to compute and analyze electronic structure and excited state adiabatic potentials of several conjugated molecules. Calculated excited state energies and parameters of molecular adiabatic surfaces characterize the coupled dynamics of vibrational and electronic degrees of freedom. The analysis identifies the specific torsional and bond-stretching nuclear motions that dominate the excited state relaxation and lead to self-localized excitations. This approach is an inexpensive and numerically efficient method of computing molecular excited state adiabatic surfaces and modeling femto-to-pico second time-dependent photoexcitation processes along chosen trajectories.
\end{abstract}

Keywords: Excited state molecular dynamics; Collective electronic oscillator method; Conjugated and donoracceptor molecules; Self-localized exciton

\section{INTRODUCTION}

Conjugated molecules have attracted significant attention due to their electronic and optical properties associated with delocalized $\pi$ electrons which make them suitable materials for various device applications (Schon et al., 2000, 2001). Single molecule photoexcitation dynamics, formation of polarons, polaritons, exciton trapping, and quasiparticle transport are important electronic phenomena which are present in these systems but challenging to a theoretical description (Tretiak et al., 1997; Heeger et al., 1988; Kirova et al., 1999). The difficulties arise from two principal features of the electronic structure: electron-electron interactions (electronic correlations) and electron-phonon interactions (vibronic coupling). Even though these features have been recognized many years ago (Heeger et al., 1988), and an enormous amount of experimental and theoretical work has been done (McBranch et al., 1997; Tretiak et al., 1997; Kirova et al., 1999; Brédas et al., 1999) the quantitative description of such processes is yet to be achieved.

*Corresponding author. E-mail: serg@lanl.gov 
One-dimensional semiconductor band models and simple tight-binding Hamiltonian approaches allow the identification of fundamental electronic features of conducting polymers (Heeger et al., 1988; Kirova et al., 1999). On the other hand, quantum chemical methods have been limited, due to a high demand of computational resources, to the treatment of excited states surfaces in very small molecules (Ben Nun et al., 2000) or to single-point computations, which compute a snapshot of the excited state structure for a given molecular geometry (Brédas et al., 1999).

Here we analyze excited state time-dependent processes in large molecular systems using the quantum-chemical semiempirical approximations. Our Excited State Molecular Dynamics (ESMD) method satisfactorily accounts for electronic correlations and allows us to model picosecond dynamics of quite large (100-200 atoms) molecular systems taking into account all $(3 N-6)$ vibrational degrees of freedom $(N$ being the number of atoms in the molecule).

\section{COMPUTATIONAL METHOD}

The excited state energy surface $E_{e}(\mathbf{q})$ as a function of nuclear coordinates $\mathbf{q}$ is computed by simply adding the excitation energy $\Omega(\mathbf{q})$ to the ground state adiabatic surface $E_{g}(\mathbf{q})$, as illustrated in Fig. 1. The ground state energy is obtained from Hartree-Fock calculations (Szabo et al., 1989). To compute the excitation energy we use the recently developed collective electronic oscillator (CEO) algorithm which combines a time-dependent Hartree Fock (TDHF) formalism with very efficient Krylov space numerical algorithms for diagonalization of the two-particle Liouville operator (Tretiak et al., 1996). As a result, the CEO computation of an excited state manifold is not substantially more numerically demanding than ground-state calculations. This allows the calculations of optical properties in large molecules (hundreds of heavy atoms). In addition, the CEO results permit the analysis of optical spectra in terms of charge distributions in excited states and motions of electrons and holes in real space (Mukamel et al., 1997). The CEO method has been successfully applied to

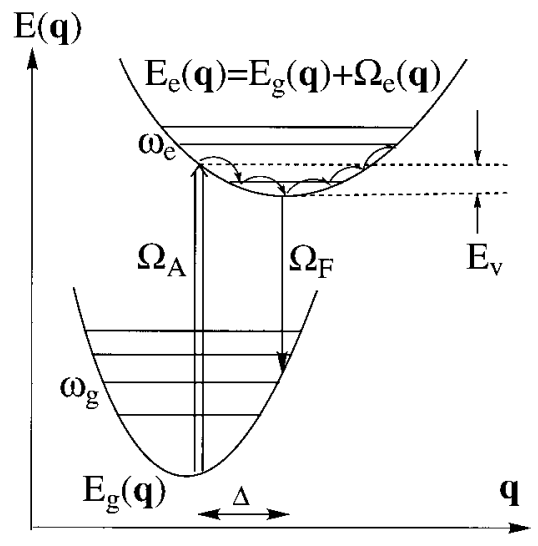

FIGURE 1 Schematic representation of ESMD propagation. Quantities of interest are: excited state energy $E_{e}(\mathbf{q})$ as a function of nuclear coordinates $\mathbf{q}$, displacements $\Delta$, curvatures $\omega_{e} / \omega_{g}$, vibrational reorganization energy $E_{v}$, vertical absorption $\Omega_{A}$ and fluorescence $\Omega_{F}$ frequencies. 
calculate optical properties in both conjugated molecules and molecular aggregates (Tretiak et al., 1996, 1997, 2000).

An essential part of the CEO method is the utilization of semiempirical models which significantly reduces the computational demand. These models complement the highly accurate $a b$ initio and density functional theory (DFT) methods; the latter are computationally expensive and applicable to a limited number of small molecules. Semiempirical approaches are based on simplified Hamiltonians, which allow efficient yet accurate computation of molecular properties. Versatile computational packages such as MOPAC and ZINDO, which use these semiempirical methods, have been developed. MOPAC, for example, is based on the AM1, PM3 and MNDO Hamiltonians that were fitted to reproduce ground state geometries, energies, and heats of formation (Dewar et al., 1985; Stewart, 1989). On the other hand, the INDO/S Hamiltonian in ZINDO was parameterized to reproduce vertical excitation energies of small organic molecules combined with the configuration interaction singles (CIS) approximation for many-electron wave-functions (Ridley et al., 1973).

Accurate and efficient calculations of excited state energy surfaces (see Fig. 1) requires a treatment of both ground state potential and excitation energies within the same Hamiltonian. We recall that, historically, Hamiltonians for the ground state (such as AM1 and PM3) were seldom applied to the excited state calculations, whereas it is known that Hamiltonians that reproduce excited state energies (such as INDO/S and CNDO/S) fail for the ground state. We therefore conducted a study of the applicability of models normally used for the ground state (AM1, PM3 and similar types) for excited state calculations. The results of computations of several conjugated molecules showed that the Hamiltonians designed for the ground state (AM1 and PM3) reproduce well the vertical excitation energies with accuracy comparable to approaches specifically fitted to the excited states (INDO/S). Figure 2 shows the structures of several donor-acceptor molecules studied in a search for large nonlinear optical responses, and the average deviation of the computed lowest state excited state (band-gap) energies from experimental values (Alain et al., 1999) for different semiempirical models. The agreement with experiment is very good, and furthermore it significantly improves when the solvation effects are taken into account (Fig. 2) (Tretiak et al., 2001). The reasonable performance of those Hamiltonians for excitation energies, combined with the fact that they were parameterized to reproduce experimental geometries, is sufficiently encouraging that we have began studying issues associated with excitation/lattice dynamics.

To conduct ESMD simulations we solve the classical equation of motion for the nuclear degrees of freedom

$$
M_{\alpha} \frac{\partial^{2} \mathbf{q}_{\alpha}}{\partial t^{2}}+\beta \frac{\partial \mathbf{q}_{\alpha}}{\partial t}=\mathbf{F}_{\alpha}=-\frac{\partial E}{\partial \mathbf{q}_{\alpha}}
$$

along the trajectory on the excited state molecular potential surface using a numerical Verlet-type algorithm (Allen et al., 1987). Here $\mathbf{q}_{\alpha}$ and $M_{\alpha}$ represent coordinates and masses of $3 N-6$ molecular vibrational normal modes and $E=E_{k}+E_{e}$ is the excited state molecular energy, $E_{\mathrm{k}}$ being the nuclear kinetic energy. The forces $\mathbf{F}_{\alpha}$ on the right-hand side of this equation are obtained by numerical differentiation. The computations could follow excited state molecular dynamics in the gas phase with vanishing 


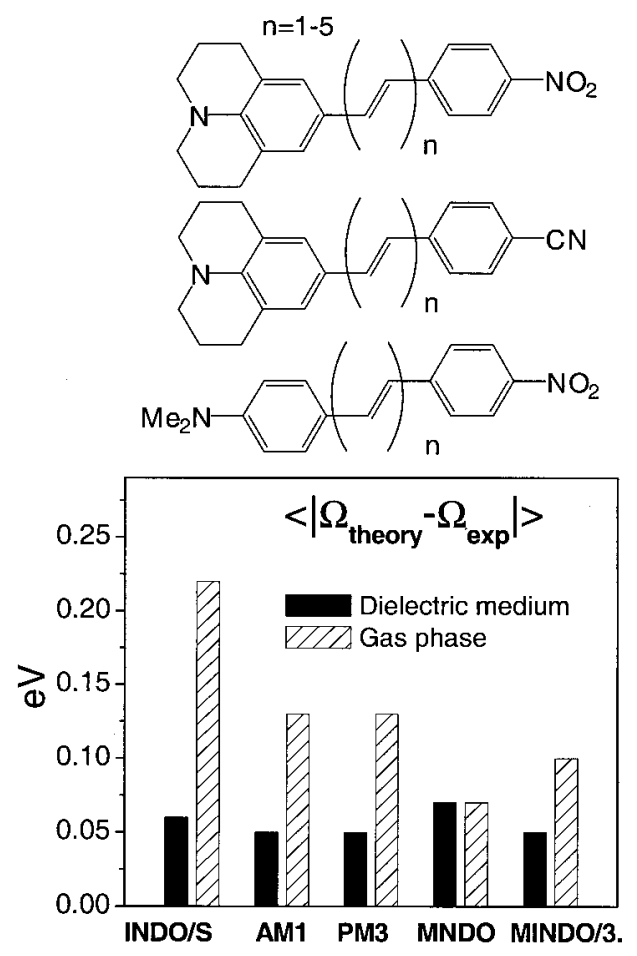

FIGURE 2 Top: structures of donor-acceptor substituted diphenyl-polyene oligomers; Bottom: deviations of magnitudes of computed vertical excitation energies from the corresponding experimental values averaged over all molecules (Alain et al., 1999; Tretiak et al., 2001).

damping $(\beta=0)$ or motion in an effective viscous medium $(\beta \neq 0)$. The latter leads to the excited state optimal geometry, as illustrated in Fig. 1.

\section{RESULTS AND DISCUSSION}

To illustrate the ESMD principles we start with a simulation of photoisomerization of hexatriene (Fig. 3). Photoisomerization is an essential photochemical reaction in nature. This excited-state photon-less relaxation leads to dramatic changes in molecular geometry (such as trans-to-cis conformation) (Baskin et al., 1996; Ben Nun et al., 2000). An important example is photoisomerization of retinal, which is the primary process in human vision. The ESMD studies show that the hexatriene being photoexcited evolves for approximately $200 \mathrm{fs}$ on the excited state surface and then undergoes rapid photoisomerization, in agreement with spectroscopic observations. In contrast, the modeling with damping allows us to find a shallow minimum for the excited state (Myers et al., 1989).

Hexatriene can be treated with more accurate first principle approaches. However, these methods are rarely applied to larger molecules because of an enormous increase of computational effort. The semiempirical ESMD still remains viable to compute such systems as, for example, donor-acceptor molecules (containing electronwithdrawing and electron-donating groups connected by a conjugated bridge), which 


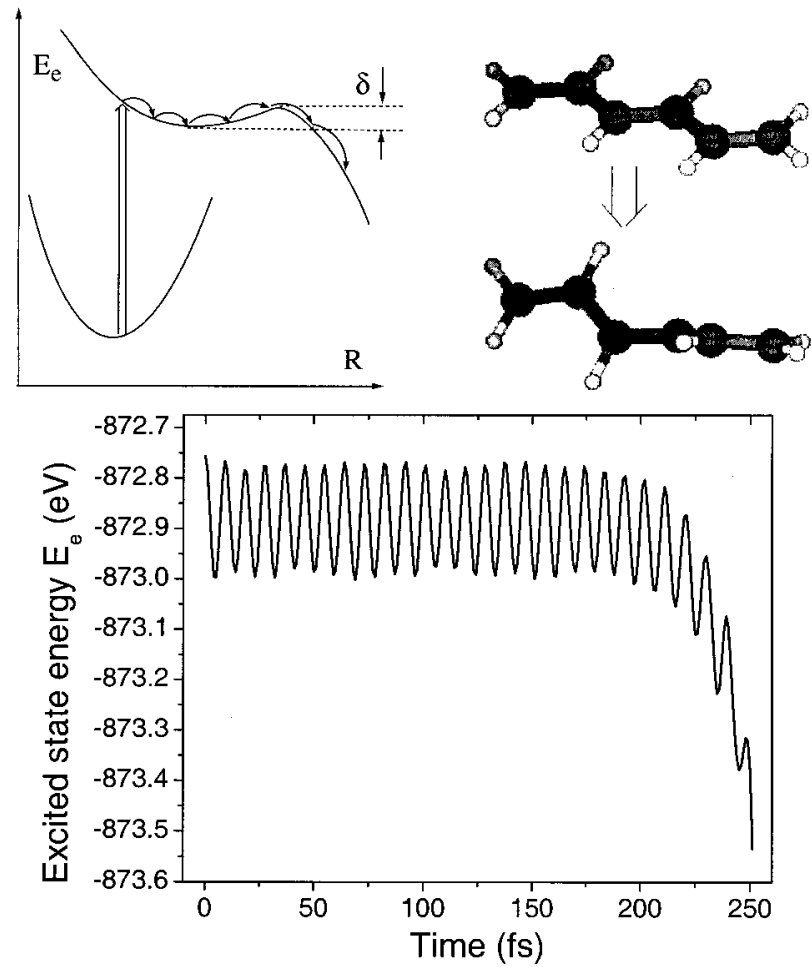

FIGURE 3 ESMD simulations of hexatriene photoisomerization with AM1 model.

undergo interesting photochemical reactions such as intramolecular relaxation involving long-range electron transfer and molecular conformation, and featuring large nonlinear responses (Tretiak et al., 2001). The ground-state optimal geometry of the donor-acceptor molecule shown in Fig. 4 calculated with the AM1 method has the structure where the neighboring phenyl-vinyl groups are twisted. This deviation from the planar geometry reduces $\pi$-electron delocalization and, therefore, the longrange charge transfer in the ground state. This situation is reversed in the excited state. The ESMD modeling shows that excited state molecular relaxation leads to the reduction of the torsional angle ("straightening out" the molecule, see Fig. 4) and to a significant redistribution of electronic charges because of enhanced delocalization of $\pi$-electron.

This strong coupling between electronic and vibrational degrees of freedom is also very important in conducting polymers. In these cases, it leads to formation of polarons, solitons, and self-trapped excitons, as was shown in early studies of polyacetylene (Heeger et al., 1988). The ESMD can be applied directly to model such processes. We study two long oligomers of polyacetylene (Fig. 5) with lengths of 30 and 60 repeat units (60 and 120 carbons, respectively) with ESMD to find the optimal geometry of the $1 B_{u}$ (band-gap) state. A prominent feature of the polymer's structure is the pattern of alternating single-like and double-like carbon-carbon bonds due to Peierls distortion of the $\pi$ electronic density, which can be characterized by the bond-length alternation (BLA) parameter (the difference between neighboring bond-lengths, see Fig. 5) (Brédas et al., 1999). This parameter is constant along the 

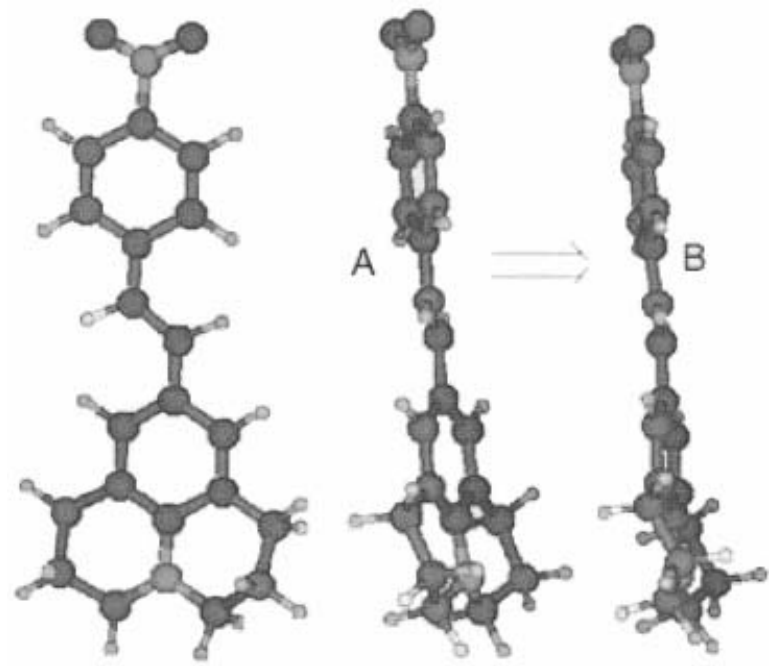

FIGURE 4 ESMD geometry relaxation along the excited state surface in a donor-acceptor compound (Fig. 2) from an initial structure (A) to an excited state optimal geometry (B) modeled with AM1. The excited state structure shows reduced torsional angle (nearly planar geometry).

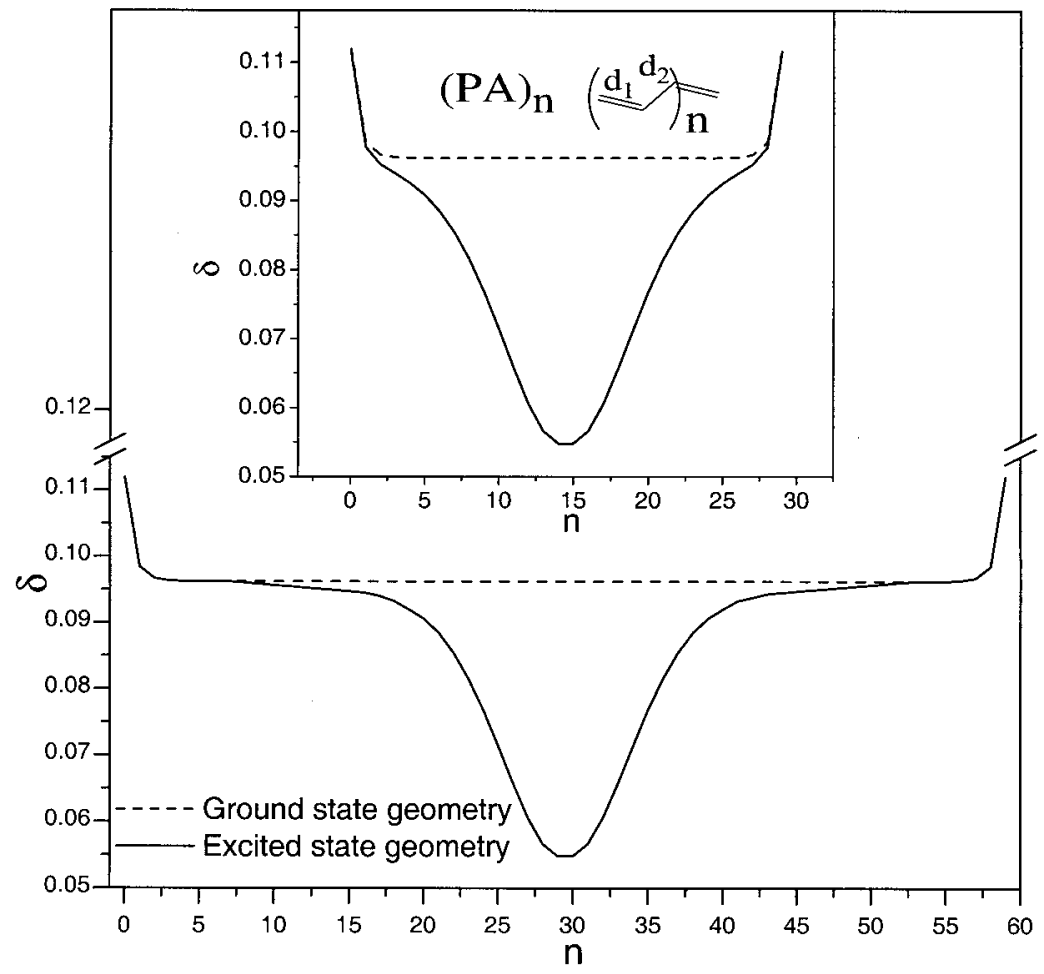

FIGURE 5 ESMD geometry relaxation along the excited state surface from the ground to the $1 B_{u}$ excited state equilibrium geometry of polyacetylene oligomers simulated with the AM1 model. The excited state structure shows a reduced bond length alternation $\delta=d_{2}-d_{1}$ in the middle of the molecule, as compared to the ground-state geometry. The size of the region with reduced bond-length alternation of about 20 repeat units is the same for $n=30$ and $n=60$ oligomer lengths. 
chain with weak end effects when the molecule is in the ground state. Relaxation of the band-gap photoexcitation leads to considerable changes in the BLA pattern. In the shorter oligomer ( $n=30$ repeat units) the BLA is reduced in the middle of the chain as shown in the inset of Fig. 5. This could be rationalized by noticing that the absorbed photon increases the energy of the $\pi$-electron system which, as a result, becomes less dimerized on the double bonds. Computation of the optimal geometry of the longer polyacetylene oligomer ( $n=60$ repeat units) results in the BLA variation shown in Fig. 5. We notice that in the regions close to the molecular ends ( $\sim 15$ repeat units) the BLA is nearly equivalent to that of the ground state. However, in the middle of the molecule $(\sim 20$ repeat units) the BLA pattern is close to that of the oligomer with $n=30$ repeat units. This manifests the process of self-trapping of the exciton on about 20 repeat units $(\sim 30 \AA)$ in the region of reduced BLA in the course of the excited state relaxation, whereas the rest of the molecule is not affected by the presence of the excitation and has the ground state geometry and distribution of the electronic density.

We conclude that the semiempirical ESMD makes possible the computation of excited state adiabatic potential surfaces and time-dependent photoexcitation processes in large molecular systems which are not possible to calculate from conventional first principle approaches. The analysis of the ESMD results reveals the principal phenomena of electron-nuclear dynamics such as electronic coupling to the torsional and bond-stretching degrees-of-freedom and trends toward planar structures and reduced BLA for the excited state optimal geometries as compared to that of the ground state. In addition, the computational results provide us with quantitative predictions such as the size of the self-trapped exciton $(\sim 30 \AA)$, and accompanying magnitudes of vibrational relaxation, Stokes shift, absorption and fluorescence spectra; these are amenable to experimental test. Applications of the ESMD method to other complex molecules will be reported elsewhere, including photoluminescent polymers, substituted oligomers, organic dyes, biological systems, etc (Tretiak et al., 2002).

\section{Acknowledgements}

This work was performed under the auspices of the US Department of Energy and the LDRD program at LANL. The numerical computations were performed using the resources of the Los Alamos Center for Nonlinear Studies.

\section{References}

Alain, V., Rédoglia, S., Blankchard-Desce, M., Lebus, S., Lukaszuk, K., Wortmann, R., Gubler, U., Bosshard, C. and Günter, P. (1999). Elongated push-pull diphenylpolyenes for nonlinear optics: molecular engineering of quadratic and cubic nonlinearities via tuning of intermolecular charge transfer. Chem. Phys., 245, 51.

Allen, M.P. and Tildesley, D.J. (1987). Computer Simulation of Liquids. Clarendon Press, Oxford.

Baskin, J.S., Banares, L., Pedersen, S. and Zewail, A.H. (1996). Femtosecond real-time probing of reactions. Dynamics of twisting, alignment, and IVR in the trans-stilbene isomerization reaction. J. Chem. Phys. A, 100, 11920.

Ben Nun M., Quenneville, J. and Martinez, T.J. (2000). Ab initio multiple spawning: photochemistry from first principles quantum molecular dynamics. J. Chem. Phys. A, 104, 5161.

Brédas, J.L., Cornil, J., Beljonne, D., Santos, D.A.D. and Shuai, Z. (1999). Excited-state electronic structure of conjugated oligomers and polymers: a quantum-chemical approach to optical phenomena. Acc. Chem. Res., 32, 267. 
Dewar, M.J.S., Zoebisch, E.G., Healy, E.F. and Stewart, J.J.P. (1985). The development and use of quantummechanical molecular-models. AM1: a new general-purpose quantum-mechanical molecular-model. J. Am. Chem. Soc., 107, 3902.

Heeger, A.J., Kivelson, S., Schrieffer, J.R. and Su, W.P. (1988). Solitons in conducting polymers. Rev. Mod. Phys., 60, 781.

Kirova, N., Brazovskii, S. and Bishop, A.R. (1999). A systematic theory for optical properties of phenylenebased polymers. Synthetic Metals, 100, 29.

McBranch, D.W. and Sinclair, M.B. (1997). In: Sariciftci, N.S. (Ed.), The Nature of the Photoexcitations in Conjugated Polymers. World Scientific Publishing, Singapore.

Mukamel, S., Tretiak, S., Wagersreiter, Th. and Chernyak, V. (1997). Electronic coherence and collective optical excitations of conjugated molecules. Science, 277, 781.

Myers, A.B. and Pranata, K.S. (1989). Excited state geometry and dynamics of trans-Hexatriene: a resonance Raman intensity study. J. Phys. Chem., 93, 5079.

Ridley, J. and Zerner, M.C. (1973). An intermediate neglect of differential overlap technique for spectroscopy: pyrrole and the azines. Theor. Chim. Acta, 32, 111.

Schon, J.H., Dodabalapur, A., Kloc, C. and Batlogg, B. (2000). A light-emitting field-effect transistor. Science, 290, 963.

Schon, J.H., Kloc, C., Hwang, H.Y. and Batlogg, B. (2001). Josephson junctions with tunable weak links. Science, 292, 252.

Stewart, J.J.P. (1989). Optimization of parameters for semiempirical methods. J. Comp. Chem., $10,210$.

Szabo, A. and Ostlund, N.S. Modern Quantum Chemistry: Introduction to Advanced Electronic Structure Theory. McGraw-Hill, New York, 1989.

Tretiak, S., Chernyak, V. and Mukamel, S. (1996). Chemical bonding and size-scaling of nonlinear polarizabilities of conjugated polymers. Phys. Rev. Lett., 77, 4656.

Tretiak, S., Chernyak, V. and Mukamel, S. (1997). Two-dimensional real-space analysis of optical excitations in acceptor-substituted carotenoids. J. Am. Chem. Soc., 119, 11408.

Tretiak, S., Saxena, A., Martin, R.L. and Bishop, A.R. (2000). Interchain electronic excitations in poly-phenylenevinylene (PPV) aggregates. J. Phys. Chem. B, 104, 7029.

Tretiak, S., Saxena, A., Martin, R.L. and Bishop, A.R. (2001). CEO/semiempirical calculations of static nonlinear polarizabilities in conjugated molecules. J. Chem. Phys., 115, 699.

Tretiak, S., Saxena, A., Martin, R. L. and Bishop, A. R. (2002). Conformational dynamics of photoexcited conjugated molecules. Phys. Rev. Lett., in press. 